\title{
Cleavable Affinity Purification (Cl-AP): A One-step Procedure to Affinity Purify Protein Complexes
}

Ammarah Tariq, Lucy Green, Christian Soeller and James G. Wakefield*

Living Systems Institute, University of Exeter, Stocker Road, Exeter, EX4 4QD, UK

*For correspondence: j.g.wakefield@exeter.ac.uk

[Abstract] Cleavable Affinity Purification (Cl-AP) uses a tripartite system of Protein-A-Streptavidin beads and nanobodies, coupled with a biotinylated, thiol-cleavable linker, providing one-step affinity purification from lysates of tissues expressing tagged proteins. This technique allows fluorescent versions of mitotic protein complexes to be isolated intact from cells, for use in biophysical and microscopy-based assays, overcoming the traditional limitations of reductionist approaches. We have used this technique successfully to purify both GFP-tagged and mCherry-tagged proteins, and their interacting partners, expressed in Drosophila melanogaster embryos. Although we demonstrate the efficacy of the GFP-binding protein and RFP-binding protein nanobodies from Chromotek, in theory any antibody could be coupled to the beads and used as a Cl-AP reagent.

Keywords: Drosophila melanogaster, Affinity purification, GFP-tag, Cell biology, Mitosis

[Background] Many proteins elicit their cellular function as part of multi-protein complexes. In order to gain full understanding of the role of a protein complex, in vivo approaches such as perturbation of protein levels/activity or monitoring dynamic localization need to be combined with in vitro biochemical assays and functional reconstitution. This holistic approach is currently severely limited. In vitro studies generally use proteins that have been individually expressed and purified in non-autogenous systems such as bacteria and insect cells, where folding and post translational modifications crucial to function may be dissimilar to that found in the original cell. Conversely, purification of specific proteins or complexes from cells/tissues generally relies on either co-immuno-precipitation or purification of a tagged version of the protein of interest which has been introduced into cells, for example using haemagglutinin (HA), FLAG ${ }^{3}$ or tandem affinity purification (TAP)-tagging. There are two main issues with these in vivo approaches: (i) the beads themselves bind to non-specific proteins leading to contamination problems, (ii) the strong antibody-antigen or tag-matrix interaction cannot be disrupted without disrupting the interactions between protein complex subunits. Modified protocols, in which the antibody-antigen interactions are disrupted using peptide competition or in which the tag is cleaved using proteases can potentially result in the purification of soluble, intact protein complex from cells. However, the former requires extensive knowledge of the antibody-antigen interface while both procedures are undertaken in non-physiological conditions and result in protein complex that requires further purification (separating the excess peptide or the protease respectively), complicating this type of approach.

We sought to develop a quick, easy and widely applicable technique that would solve these problems- 
allowing the isolation of a protein complex of interest in soluble form, in the buffer of choice. We chose GFP as our affinity tag, given the added advantage of being able to assess the dynamic localization and functionality of the fusion protein within a cell or tissue, prior to isolation. We term this technique Cleavable Affinity Purification (Cl-AP).

\section{Materials and Reagents}

1. $15 \mathrm{ml}$ conical tube

2. Beckman tube (Beckman Coulter, catalog number: 343776)

3. Pipette tip with a diameter of $0.6 \mathrm{~mm}$ (e.g., Gel-Saver ${ }^{\circledR}$ II, STARLAB, catalog number: I10220600)

4. Spin Desalting Column, $2 \mathrm{ml}, 10$ columns, for 200-700 $\mu$ l samples, 7,000 MWCO (Zeba ${ }^{\mathrm{TM}}$, catalog number: 89882)

5. Tissue of choice (e.g., Drosophila embryos)

6. GFP VHH, recombinant binding protein (Chromotek, catalog number: gt-250)

7. $1 \mathrm{x}$ Phosphate-buffered Saline (PBS): $0.1 \mathrm{M}$ sodium phosphate, $0.15 \mathrm{M} \mathrm{NaCl}, \mathrm{pH} 7.2$

8. Sulfo-NHS-SS-Biotin (Thermo Scientific, catalog number: 21945)

9. Anhydrous dimethylformamide (DMF) (Sigma-Aldrich, catalog number: 227056)

10. Buffer of choice (e.g., $1 \times$ BRB80: $80 \mathrm{mM}$ PIPES, $1 \mathrm{mM} \mathrm{EGTA}, 1 \mathrm{mM} \mathrm{MgCl}_{2}$, pH 6.9 at $4{ }^{\circ} \mathrm{C}$ )

11. IGEPAL (Sigma, catalog number: I8896)

12. Phenylmethylsulfonyl fluoride (PMSF) (Sigma, catalog number: 10837091001/PMSF-RO)

13. Pi (cOmplete Mini protease inhibitor) (Roche, catalog number: 11836170001) Working concentration: 1 tablet per $10 \mathrm{ml}$ extraction solution

14. PPi (PhosSTOP phosphatase inhibitor) (Roche, catalog number: 4906837001) Working concentration: 1 tablet per $10 \mathrm{ml}$ extraction solution

15. High Capacity Streptavidin Agarose Resin (Pierce, catalog number: 20349)

16. Dithiothreitol (DTT) (Thermo scientific, catalog number: R0861)

17. Biotinylated antibody/nanobody (Chromotek, catalog number: gt-250)

18. Liquid nitrogen

\section{Equipment}

1. Optima MAX-TL ultracentrifuge with TLA-120.1 rotor (Beckman Coulter)

2. Microcentrifuge

3. Rocking platform shaker

4. Rotating disc mixer

5. Dounce homogenizer

6. $-80^{\circ} \mathrm{C}$ freezer 


\section{Procedure}

A. Preparation of GFP-TRAP-Sulfo beads

Desalt/Buffer Exchange of the nanobody (e.g., GFP-binding-protein)

Note: The nanobody must be completely desalted into $1 x$ PBS before being modified with SulfoNHS-SS-Biotin.

Zeba $^{\mathrm{TM}}$ Spin desalting columns are used according to manufacturers instructions .

1. Remove column's bottom closure, loosen the cap, and place into a $15 \mathrm{ml}$ conical tube.

2. Centrifuge at $1,000 \times g$ for $2 \mathrm{~min}$ in a benchtop microfuge. Discard the flow-through (to remove storage buffer).

3. Place a mark on the side of the column where the compacted resin is slanted upward. Place column in the microcentrifuge with the mark facing outward in all subsequent centrifugation steps.

4. Equilibrate the Zeba column with $1 \mathrm{ml} 1 \times \mathrm{PBS}$. Centrifuge at 1,000 $\times \mathrm{g}$ for $2 \mathrm{~min}$ and discard the flow-through.

5. Repeat Step A4 two more times.

6. Insert the equilibrated column in a new conical tube. Apply $200 \mu \mathrm{l}$ of nanobody $(1 \mathrm{mg} / \mathrm{ml})$ directly on the filter.

7. Centrifuge at $1,000 \times g$ for 2 min and collect the flow through (nanobody).

B. Biotin-labelling Reaction

1. Allow vial of Sulfo-NHS-SS-Biotin to equilibrate to ambient temperature before opening.

2. Immediately before use, dissolve the Sulfo-NHS-SS-Biotin in DMF to give a $8 \mathrm{mM}$ solution.

3. Add $86.1 \mu \mathrm{l}$ of Sulfo-NHS-SS-Biotin solution to $200 \mu \mathrm{l}$ of nanobody $(1 \mathrm{mg} / \mathrm{ml})$.

4. Mix immediately by inversion.

5. Incubate on ice for $2 \mathrm{~h}$.

6. Stop the reaction by removing the excess biotin reagent using a fresh Zeba spin column (i.e., repeat Steps A1-A7).

C. Linking the Biotinylated antibody to Streptavidin Agarose beads

1. Mix the streptavidin agarose resin to ensure an even suspension.

2. Wash $40 \mu \mathrm{l}$ of bead slurry ( $20 \mu \mathrm{l}$ packed beads) by resuspension in $1 \times$ PBS, pelleting the beads at 2,500 $\times g$ for $2 \mathrm{~min}$ in a microcentrifuge. Repeat three more times.

3. Add biotinylated nanobody solution (from B6) to washed streptavidin agarose beads and allow to bind to beads using either a rocking platform shaker or a rotating disc mixer for $1 \mathrm{~h}$ at $4{ }^{\circ} \mathrm{C}$.

4. Remove any unbound antibody by washing three times as described above (C2) but using the buffer of choice (e.g., BRB80 + 0.1\% IGEPAL) instead of PBS. 
5. After removing the supernatant from the last wash, resuspend the beads in the buffer of choice, to maintain a $50 \%(40 \mu \mathrm{l})$ bead slurry. Store at $4{ }^{\circ} \mathrm{C}$.

Note: Cl-AP beads can be stored at $4{ }^{\circ} \mathrm{C}$ for at least 3 months with no loss of activity.

D. Homogenization of tissue and preparation of the High-speed Supernatant (HSS)

Drosophila embryos expressing the GFP-fusion protein of interest are collected (batches of 0- to 3h-old embryos), dechorionated, weighed, flash frozen in liquid nitrogen and stored at $-80^{\circ} \mathrm{C}$, until approximately $200 \mathrm{mg}$ has been collected. All subsequent steps are performed on ice or in a $4{ }^{\circ} \mathrm{C}$ cold room using prechilled buffers and pipettes.

Note: Alternative tissues, for example tissue culture cells transiently expressing GFP- or mCherryexpressing proteins, can be substituted.

1. Transfer the tissue to a Dounce homogenizer on ice.

2. Add $400 \mu \mathrm{l}$ of an appropriate homogenising buffer (e.g., BRB80 $+0.1 \%$ IGEPAL + PMSF $+\mathrm{Pi}$ and PPi) and homogenize.

3. Centrifuge homogenate at $14,000 \times g$ for $10 \mathrm{~min}$ in a microfuge at $4{ }^{\circ} \mathrm{C}$.

4. Transfer supernatant to a Beckman tube and spin at $100,000 \times g$ for $10 \mathrm{~min}$ at $4{ }^{\circ} \mathrm{C}$.

5. Transfer supernatant to a fresh tube and spin again for $30 \mathrm{~min}$ at 100,000 $\mathrm{xg}$.

E. Incubation of HSS with cl-AP

Transfer the HSS to a new $1.5 \mathrm{ml}$ tube and incubate with $20 \mu \mathrm{l}$ (1:1 slurry) of GFP-TRAP-Sulfo beads, overnight in cold room on rotating wheel.

F. Cleaving of Sulfo-beads

1. Wash beads with assay buffer (e.g., $1 \mathrm{ml} \mathrm{BRB} 80+0.1 \%$ IGEPAL) 4 times (Step C2) to remove unbound and non-specifically bound proteins.

2. After removing the supernatant from the last wash, add $50 \mu \mathrm{l}$ of assay buffer containing $50 \mathrm{mM}$ DTT (final concentration), to cleave the disulfide bond in the spacer arm.

3. Incubate sample in orbital shaker at RT for $2 \mathrm{~h}$.

4. Pellet the beads at $2,500 \times g$ for 2 min in a microcentrifuge.

5. Transfer the supernatant (eluate) to a fresh tube using a Gel-Saver II pipette tip.

Note: The purified protein/protein complex can now be used directly for in vitro assays. If $50 \mathrm{mM} \mathrm{DTT}$ is found to interfere with a particular assay, it can be eliminated using a desalting column (Steps A1-A7).

\section{Acknowledgments}

This work was funded by University of Exeter (Proof of Concept), Lucy Green; University of Exeter (Carlota Palmer PhD studentship) Ammarah Tariq. 
We thank Stephen Green and Mark Wood (Exeter, UK) for initial discussions about photocleavable and other tags, and Mary Munson (Massachusetts, US) for highlighting the potential of thiol-cleavable tags for native complex purification, as published by the Rout lab (Rockefeller, US) (Fridy et al., 2015). We thank Chromotek for the gift of GFP-binding protein; Jack Chen, Dan Hayward and Chris Sullivan for initial attempts to develop the Cl-AP technique.

The original research article where this protocol was used is Tariq et al., 2020.

\section{Competing interests}

No competing interests declared.

\section{$\underline{\text { References }}$}

1. Fridy, P.C., Thompson, M.K., Ketaren, N.E. and Rout, M.P. (2015). Engineered high-affinity nanobodies recognizing staphylococcal Protein $A$ and suitable for native isolation of protein complexes. Anal Biochem 15: 477:92-4.

2. Tariq, A., Green, L., Jeynes, J. C. G., Soeller, C. and Wakefield, J. G. (2020). In vitro reconstitution of branching microtubule nucleation. Elife 9: e49769. 